\title{
Diquarks: a QCD sum rule perspective
}

\author{
Kyungil Kim, ${ }^{1}$ Daisuke Jido, ${ }^{2}$ and Su Houng Lee ${ }^{1,2}$ \\ ${ }^{1}$ Institute of Physics and Applied Physics, Yonsei University, Seoul 120-749, Korea \\ ${ }^{2}$ Yukawa Institute for Theoretical Physics, Kyoto University, Kyoto 606-8502, Japan
}

(Dated: December 14, 2017)

\begin{abstract}
We propose a phenomenological QCD sum rule with an explicit diquark field to investigate the essential ingredients inside the hadrons. By introducing the mass $m_{\phi}$ and the condensate $\left\langle\phi^{2}\right\rangle$ for the diquark field as parameters in the model, we find that the sum rule works well for $\Lambda, \Lambda_{c}$ and $\Lambda_{b}$. This implies that these $\Lambda$ baryons can be represented by a diquark and a quark configuration. We also find that there is a duality relation among the parameters $\left(m_{\phi},\left\langle\phi^{2}\right\rangle\right)$ for which the sum rule is equally good. In the limit when $\left\langle\phi^{2}\right\rangle=0$, we find $m_{\phi}=400 \mathrm{MeV}$, which can be thought of as the constituent diquark mass.
\end{abstract}

PACS numbers: 14.40.Gx,11.55.Hx,24.85.+p

\section{INTRODUCTION}

Quests for fundamental correlations in strongly interacting systems can be a clue to understanding the structure of the physical systems. For instance, the Cooper pair, which is a correlated electron pair, is a key concept to understand many-electron systems and leads to superconductivity after it condenses with other pairs. Also in nuclear physics, two-nucleon pair correlation can be an important degree of freedom to describe the nucleus, such as in the interacting boson model. In hadron physics, two-quark pair correlations, or diquarks, were introduced in the early days of quark model to explain systematics in baryon spectroscopy [1, 2]. Over the years, experimental findings in the structure function, fragmentation function and weak decay processes pointed to the existence of a strong diquark correlation, especially in a definite spinflavor configuration, which is the so-called good diquark channel [3].

In two-color QCD with massless quarks, interquark interactions have exactly the same strength as $\bar{q}-q$ correlations due to the Pauli-Gürsey symmetry. This symmetry is explicitly broken in three-color QCD, but the remnant is seen in the good diquark channel, which has a resemblance but less strong attraction to its counterpart of $\bar{q} q$. The strong diquark correlation in the good channel can be derived from one gluon exchange potential or through the instanton-induced interaction, and is found in the lattice calculation in the Landau gauge 4] and Coulomb gauge [5]. It is also found in the configuration with an additional color source to make the whole configuration gauge invariant [6, 7]. However, in most hadron configurations the strong diquark correlation expressible as an elementary constituent has not been so evident. The reason why diquarks cannot be treated as an elementary constituent can be traced back to the original work by Jaffe on the tetraquark picture for the scalar nonet [8]. There, the wave function for $\sigma$ is found to be dominantly composed of the $\pi-\pi$ state in the meson-meson basis, which translates into a small diquark-antidiquark component in their respective good channels. This means that when an antiquark is present, the diquark would break and form a quark-antiquark pair, which is energetically more favorable than a diquark. This phenomenon is related to the reason we have quark condensate over diquark-antidiquark condensate in the QCD vacuum.

Nonetheless, there are certain configurations where the good diquark correlation is the strongest and its role is important. The color superconducting phase appearing at high density is an example [9, 10]. There the strong correlation in the good diquark channel is responsible for the Cooper pairing. At the hadron level, the simplest configuration of this sort is the baryon composed of a diquark $(u d)$ and a spectator quark $(h)$; namely $\Lambda_{h}$, where $h$ can be $s, c$ or $b$ quarks. The reason that a diquark correlation remains strong can be understood from the colorspin interaction, which is known to be inversely proportional to the constituent mass of the quarks. Inside the $\Lambda$, the color-spin interaction between $u-h$ quarks cancels that between $d-h$ quarks when the diquark $(u d)$ is in the $\mathrm{I}=0$ channel. Moreover, the color-spin interactions with the heavy quark itself become smaller as $h$ becomes heavy 11]. Hence the diquark will retain its strong correlation with small $u h$ or $d h$ correlations. Other configurations, where diquark correlations are expected to remain important are hadrons with large angular momentum [12] and exotic configurations with heavy quarks [13 15].

In this paper, we will show that, inside the $\Lambda_{h}$, diquark correlation is indeed strong so that the diquark can be treated as an elementary constituent. We further show that one can extract well-defined parameters for the $d i$ quark, from which one can also define the constituent diquark mass.

\section{FORMULATION}

To carry out our task, we propose a framework for describing the $\Lambda_{h}$ based on the QCD sum rule technique [16, 17]. In the usual study of baryon spectra in the QCD sum rule, one calculates a correlation function of baryonic currents composed of the quark fields using the operator product expansion [18]. If the correlation of 
a pair of quarks is strong, the pair can be regarded effectively as elementary degrees of freedom. Thus, we introduce an explicit scalar-diquark field $\phi_{a}^{\dagger}$ with the color charge $a$ in $\overline{3}$ and calculate the correlation function of the $\Lambda_{h}$ current given by $J_{\Lambda}=\phi_{a}^{\dagger} h^{a}$ with $h$ being the heavy quark field:

$$
\begin{aligned}
\Pi(q) & =i \int d^{4} x e^{i q x}\left\langle T\left[J_{\Lambda}(x), \bar{J}_{\Lambda}(0)\right]\right\rangle \\
& =\hat{q} \Pi^{e}\left(q^{2}\right)+\Pi^{o}\left(q^{2}\right),
\end{aligned}
$$

where $\hat{q} \equiv q^{\mu} \gamma_{\mu}$.

The dynamics of the diquark field obeys the color $\mathrm{SU}(3)$ gauge theory:

$$
L_{\phi}=\phi^{\dagger}\left[D^{2}+m_{\phi}^{2}\right] \phi
$$

where the covariant derivative for the diquark is given by $D_{\mu}=\partial_{\mu}-i g t^{a} A_{\mu}^{a}$ and $m_{\phi}$ is the diquark mass. We incorporate Eq. (2) into the QCD Lagrangian. As we emphasized before, we will be using this Lagrangian only in configurations where the diquark correlations remain strong. One should note that the high energy limit of the correlation in Eq. (1D) does not have a corresponding high-energy limit in real QCD, as the diquark field is treated here as an elementary field, which is not the case in reality. Nevertheless, one can use this framework to check the validity of the description of $\Lambda_{h}$ by confirming that the current $J_{\Lambda}$ couples strongly to the physical $\Lambda_{h}$ at low energy and that the correlation function reproduces the correct mass of $\Lambda_{h}$.

The operator product expansion (OPE) makes it possible to write down the correlation function in terms of vacuum expectation values of the elementary fields stemming from nonperturbative dynamics. The OPE of both the even and odd part of the correlation function (1) can be calculated using the fixed-point gauge. We include the diquark condensate $\left\langle\phi^{\dagger} \phi\right\rangle \equiv\left\langle\phi^{2}\right\rangle$ and perform the calculation to leading order in $\alpha_{s}$ up to terms of condensates having dimension 6 . Note that since the diquark field has dimension 1 in the $\Lambda_{h}$ current $J_{\Lambda}=\phi^{\dagger} h$, the correlation function (10) has mass dimension 1 , which is smaller than the usual baryonic correlators.

The perturbative part is as follows:

$$
\begin{aligned}
& \operatorname{Im} \Pi_{\mathrm{p}}^{e}=\frac{3 \pi}{(4 \pi)^{2}} \frac{\tilde{q}^{2}}{2 q^{2}} \frac{\bar{q}^{2}}{q^{2}} u \\
& \operatorname{Im} \Pi_{\mathrm{p}}^{o}=m_{h} \frac{3 \pi}{(4 \pi)^{2}} \frac{\bar{q}^{2}}{q^{2}} u,
\end{aligned}
$$

where $\bar{q}^{2} \equiv q^{2}-\left(m_{h}-m_{d}\right)^{2}, \tilde{q}^{2} \equiv q^{2}+m_{h}^{2}-m_{\phi}^{2}$ and $u^{2} \equiv 1-4 m_{h} m_{\phi} / \bar{q}^{2}$. It is worth noting that, although we call Eq. (3) the perturbative part that is obtained by the perturbative calculation of our effective model, since we introduce the diquark as an elementary field, these terms contain highly non-perturbative interactions in the sense of original QCD.
The condensate parts are given as

$$
\begin{aligned}
\Pi_{\mathrm{c}}^{e}= & C_{\phi}^{e}\left\langle\phi^{2}\right\rangle+C_{h}^{e}\langle\bar{h} h\rangle+\left[\frac{3 m_{\phi}^{2}}{6 \cdot 24} F_{3}^{40}-\frac{C_{\phi}^{e}}{96 m_{\phi}^{2}}\right]\left\langle\frac{\alpha}{\pi} G^{2}\right\rangle \\
& +\left[-\frac{3 m_{h}^{2}}{12^{2}} F_{3}^{13}+\frac{C_{h}^{e}}{12 m_{h}}\right]\left\langle\frac{\alpha}{\pi} G^{2}\right\rangle-\frac{m_{\phi}^{2}}{8 D_{\phi}^{3}}\langle g \bar{h} \sigma \cdot G h\rangle \\
& -\frac{1}{9}\left(\frac{2}{D_{h} D_{\phi}}+\frac{1}{D_{\phi}^{2}}+\frac{4\left(q^{2}+m_{h}^{2}\right)}{D_{h}^{2} q^{2}}\right)\left\langle g^{2} \phi^{2} \bar{h} h\right\rangle, \quad(4)
\end{aligned}
$$

where $D_{\phi} \equiv q^{2}-m_{\phi}^{2}, D_{h} \equiv q^{2}-m_{h}^{2}, F_{\ell}^{m n} \equiv \int_{0}^{1} d x\left(x^{m}(1-\right.$ $\left.x)^{n}\right) /\left(\Delta^{2}\right)^{\ell}$ with $\Delta^{2} \equiv x(x-1) q^{2}+(1-x) m_{h}^{2}+x m_{\phi}^{2}$, and

$$
C_{\phi}^{e}=-\frac{1}{4 m_{\phi}^{2} q^{4}}\left[q^{4}-\left(m_{\phi}^{2}-m_{h}^{2}\right)^{2}+2 m_{\phi}^{2} q^{2}-\tilde{q}^{2} \bar{q}^{2} u\right],
$$

$C_{h}^{e}=-\frac{m_{h}}{\left(4 m_{h}^{2} q^{2}\right)^{2}}\left(2 m_{h}^{2} q^{2}-\left(\tilde{q}^{2}\right)^{2}+\tilde{q}^{2} \sqrt{\left(\tilde{q}^{2}\right)^{2}-4 m_{h}^{2} q^{2}}\right)$,

for the even part, and

$$
\begin{aligned}
\Pi_{\mathrm{c}}^{o} & = \\
& C_{\phi}^{o}\left\langle\phi^{2}\right\rangle+C_{h}^{o}\langle\bar{h} h\rangle+\left[\frac{3 m_{h} m_{\phi}^{2}}{6 \cdot 24} F_{3}^{30}-\frac{C_{\phi}^{o}}{96 m_{\phi}^{2}}\right]\left\langle\frac{\alpha}{\pi} G^{2}\right\rangle \\
& +\left[\frac{3 m_{h}}{12^{2}}\left(-m_{h}^{2} F_{3}^{13}-m_{\phi}^{2} F_{3}^{22}+F_{2}^{02}\right)+\frac{C_{h}^{o}}{12 m_{h}}\right]\left\langle\frac{\alpha}{\pi} G^{2}\right\rangle \\
& +\frac{1}{3^{3} \cdot 2} \frac{1}{D_{\phi}^{3}}\left\langle g^{2}(\bar{h} h)^{2}\right\rangle-\frac{2 m_{h}}{9 D_{h} q^{2}}\left(\frac{1}{D_{\phi}}+\frac{4}{D_{h}}\right)\left\langle g^{2} \phi^{2} \bar{h} h\right\rangle \\
& +\frac{m_{\phi}^{2}}{D_{\phi}^{4}}\left(\frac{1}{3^{3} 2^{4}}\left\langle g^{2}(\bar{h} h)^{2}\right\rangle-\frac{m_{h}}{2^{6}} g\langle h \sigma \cdot G h\rangle\right),
\end{aligned}
$$

where

$$
\begin{aligned}
& C_{\phi}^{o}=-\frac{m_{h}}{2 m_{\phi}^{2} q^{2}}\left(q^{2}+m_{\phi}^{2}-m_{h}^{2}-\bar{q}^{2} u\right) \\
& C_{h}^{o}=-\frac{1}{8 m_{h}^{2} q^{2}}\left(\sqrt{\left(\tilde{q}^{2}\right)^{2}-4 m_{h}^{2} q^{2}}-\tilde{q}^{2}\right),
\end{aligned}
$$

for the odd part. In each part, the first contribution to the gluon condensate comes from gluon operators emanating from the diquark and the second contribution comes from the quark propagator. The factors of the gluon condensate proportional to $C_{\phi}\left(C_{h}\right)$ subtract the zero momentum part of the diquark (quark) propagator contributing to the gluon condensate, which should be taken into account through the diquark (quark) condensate. The parts to be subtracted can be obtained by the contributions from the diquark (quark) condensate after expressing the condensate in terms of the gluon condensate [19]:

$$
\begin{aligned}
\langle\bar{h} h\rangle & =-\frac{1}{12 m_{h}}\left\langle\frac{\alpha}{\pi} G^{2}\right\rangle \\
\left\langle\phi^{\dagger} \phi\right\rangle & =\frac{1}{96 m_{\phi}^{2}}\left\langle\frac{\alpha}{\pi} G^{2}\right\rangle .
\end{aligned}
$$


In fact, these equations are obtained as the leading terms of the heavy mass expansions. Therefore, for the $\Lambda_{c}, \Lambda_{b}$ sum rule, we can use Eq. (6) in Eq. (4) and in Eq. (5) and obtain their sum rule without heavy quark condensate.

Both the even and odd part of the correlation function satisfies the following Borel transformed dispersion relation:

$$
\text { B.T. } \Pi\left(q^{2}\right)=\frac{1}{M^{2}} \int_{\left(m_{\phi}+m_{h}\right)^{2}}^{\infty} d s \frac{1}{\pi} \operatorname{Im} \Pi(s) e^{-s / M^{2}},
$$

where B.T. stands for Borel transformation and $M$ is the Borel mass. After the Borel transformation, the correlation function is expressed as an expansion in $1 / M^{2}$ such that higher dimension operators have more powers of $1 / M^{2}$. The Borel transformation of the fifth term of Eq. (4) and the last term of Eq. (5) proportional to $m_{\phi}^{2}$ give contributions with a factor of $\exp \left[-m_{\phi}^{2} / M^{2}\right] / M^{8}$, which are of a much higher order in the $1 / M^{2}$ expansion. Thus we neglect these terms in our calculation.

We assume that the $\Lambda_{h}$ contribution to the correlation function in Eq. (1) is given by

$$
\Pi(q)=\frac{f\left(\hat{q}+m_{\Lambda}\right)}{q^{2}-m_{\Lambda}^{2}+i \epsilon}+\theta\left(q^{2}-s_{0}\right)\left(\hat{q} \Pi_{\mathrm{OPE}}^{e}+\Pi_{\mathrm{OPE}}^{o}\right)
$$

where $f$ is a normalization of the $\Lambda_{h}$ propagator, $\Pi_{\mathrm{OPE}}^{i=e, o} \equiv$ $\Pi_{\mathrm{p}}^{i}+\Pi_{\mathrm{c}}^{i}$ is the correlation function calculated by OPE and given in Eqs.(3), (4) and (5), and $s_{0}$ is the threshold parameter that represents the energy scale where the quark-hadron duality ansatz works in the QCD sum rule. Our theory will only comprise a limited phase space of the whole QCD. However, once we introduce the diquark field as an effective degree of freedom, the spectral density at high energy can be calculated perturbatively as asymptotic freedom remains valid also in scalar QCD.

In principle, the sum rules for the $\Lambda_{h}$ mass can be obtained by the following:

$$
m_{\Lambda}=\frac{\mathbf{B} \cdot \mathbf{T} \cdot\left[\theta\left(s_{0}-s\right) \Pi^{o}(s)\right]}{\mathbf{B} \cdot \mathbf{T} \cdot\left[\theta\left(s_{0}-s\right) \Pi^{e}(s)\right]}
$$

or

$$
m_{\Lambda}^{2}=\frac{M^{2} \frac{d}{d M^{2}} M^{2} \mathbf{B} \cdot \mathbf{T} \cdot\left[\theta\left(s_{0}-s\right) \Pi^{i}(s)\right]}{\mathbf{B} \cdot \mathbf{T} \cdot\left[\theta\left(s_{0}-s\right) \Pi^{i}(s)\right]},
$$

where $i=e$ or $o$. It should be noted however, that $\Pi^{o}$ is proportional to $m_{h}$ except for the term proportional to $\langle\bar{h} h\rangle$, and hence is dominated by a single term when $m_{h}$ is small. Therefore, $\Pi^{e}$ should not be reliable for $\Lambda$. In such a case, one should use Eq. (11) with $i=e$ to calculate the mass. Moreover, as a natural extension of the $\Lambda$ sum rule, we will use Eq. (11) with $i=e$ also for $\Lambda_{c}$ and $\Lambda_{b}$.

We use conventional values of the quark and gluon condensates and the quark mass, which were determined in other QCD sum rules. For the case of $h=$ $s$, we use $\left\langle\frac{\alpha}{\pi} G^{2}\right\rangle=(0.33 \mathrm{GeV})^{4},\langle\bar{s} s\rangle=0.8\langle\bar{q} q\rangle=$
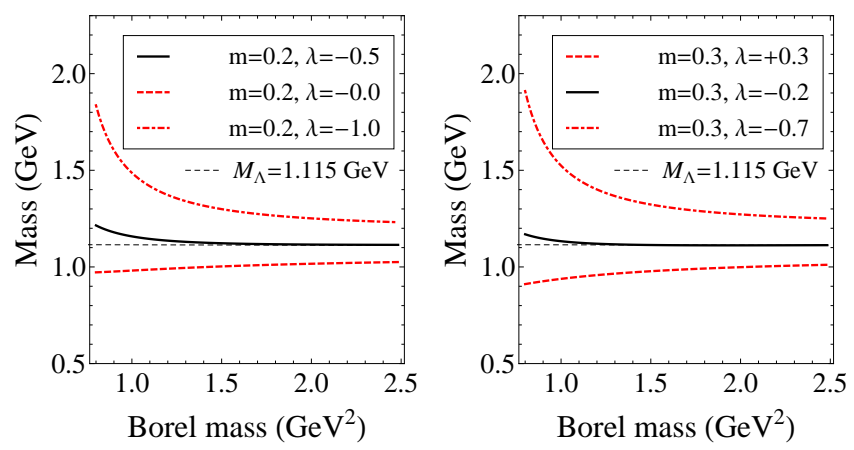

FIG. 1: (Color online) Borel curves for $\Lambda$ with several sets of paramters.

$-0.8(0.23 \mathrm{GeV})^{3}$, and $m_{s}=0.12 \mathrm{GeV}$ [22]. For the higher dimensional condensates, we adopt the vacuum saturation hypothesis. That is $\left\langle g^{2}(\bar{h} h)^{2}\right\rangle=\kappa g^{2}\langle\bar{h} h\rangle^{2}$ [20, 21], with $g=\kappa=1$. We will also show that the result with different values of $\kappa$ gives almost the same result as the sum rule has only a weak dependence on these operators. For the sum rules of $\Lambda_{c}$ and $\Lambda_{b}$, we use $m_{c}=1.47 \mathrm{GeV}$ and $m_{b}=4.6 \mathrm{GeV}$ [23], and the values of the condensates obtained by Eq. (6).

The diquark quantities $m_{\phi}$ and $\left\langle\phi^{\dagger} \phi\right\rangle$ are not determined yet and can be our model parameters to be determined within our framework. If the diquark description of $\Lambda_{h}$ is valid, the sum rule should work well and reproduce the observed $\Lambda_{h}$ mass with reasonable values of the diquark parameters. To parametrize the variation of the diquark condensate, we introduce a scaling factor $\lambda$ with respect to its value obtained in the heavy diquark mass limit given in Eq. (7) with $m_{\phi}=0.2 \mathrm{GeV}$ :

$$
\left\langle\phi^{\dagger} \phi\right\rangle=\lambda \frac{1}{96(0.2 \mathrm{GeV})^{2}}\left\langle\frac{\alpha}{\pi} G^{2}\right\rangle
$$

\section{RESULTS}

A. $\Lambda$

The plots in Fig. 1 show the Borel sum rules for the $\Lambda$ mass obtained by using $\Pi^{e}$ in Eq. (11) for diquark masses of 0.2 and $0.3 \mathrm{GeV}$ for the upper and lower figure respectively with several condensate values. One finds that for the upper curve, the $\Lambda$ mass is well reproduced with a stable Borel curve for the diquark condensate value of $\lambda=-0.5$, which corresponds to $\left\langle\phi^{2}\right\rangle=-(0.04 \mathrm{GeV})^{2}$. The fact that good Borel stability has been obtained implies that the correlation function of $J_{\Lambda}$ with the diquark field strongly couples to the low-lying $\Lambda$ baryon and thus the description of $\Lambda$ in terms of the diquark degrees of freedom works well.

The Borel window in this case is set between $M^{2}=1.3$ and $M^{2}=2.2 \mathrm{GeV}^{2}$. The lower limit is determined by 

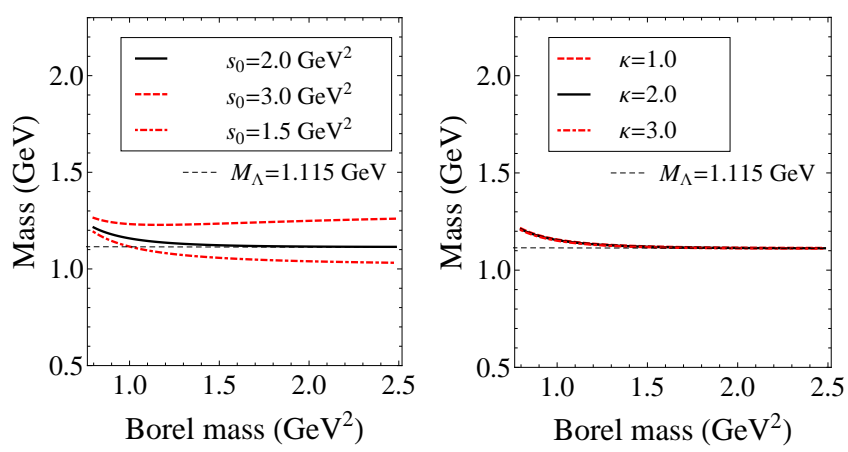

FIG. 2: (Color online) Borel curves for $\Lambda$ with different threshold paramters (left) and with different $\kappa$ (right).

the OPE convergence where the contribution of the condensate part is less than $30 \%$ of the perturbative part, while the upper limit is determined by the pole dominance in which the $\Lambda$ pole contribution exceeds the contributions coming from the second term of Eq. (9). Note that the present sum rule has a much wider Borel window and better stability than the usual baryonic QCD sum rule. The threshold $s_{0}$ was set at $2 \mathrm{GeV}^{2}$ as in a previous study [24]. The sum rule is rather stable under a small variation in the threshold parameter leading to the same diquark parameters. For larger variation, one finds that the sum rule becomes less stable, as can be seen in the left figure of Fig. 2 .

Next we discuss diquark parameter dependence of the sum rule stability. When $\lambda$ becomes smaller than -0.5 for $m_{\phi}=0.2 \mathrm{GeV}$, the Borel stability is lost and the mass monotonically decreases toward smaller Borel mass. For larger $\lambda$ the curve breaks down at lower Borel mass. Similar behavior is found for a larger diquark mass of $0.3 \mathrm{GeV}$, as seen in the right figure. On the other hand, this time, the stable curve is obtained when the diqurk condensate is smaller $\lambda=0.2$. One can further analyze the sum rule for different values of the diquark mass and diquark condensate and find a strong correlation between the two parameters. That is to say, to reproduce the physical $\Lambda$ mass with a stable Borel curve, for larger (smaller) diquark mass, we need smaller (larger) diquark condensate. In general, obtaining a stable curve should have enough information to determine a unique pair of the parameters, since it is not trivial at all that the sum rule works well with the Borel stability. Nevertheless, in the present case, we find a duality-type relation among the two parameters. This means that the $\Lambda$ mass may be essentially controlled by a single physical quantity, such as a "constituent" diquark mass. In the limit when the diquark condensate is zero $(\lambda=0)$, one finds that the necessary diquark mass is around $0.4 \mathrm{GeV}$, which corresponds to the constituent diquark mass.

Finally, we show the sum rule with $\left(\lambda, m_{\phi}\right)=$ $(-0.5,02 \mathrm{GeV})$ but with different $\kappa$. As can be seen in the right figure of Fig. 2, the dashed and dot-dashed lines are almost indistinguishable from the solid line, suggest-
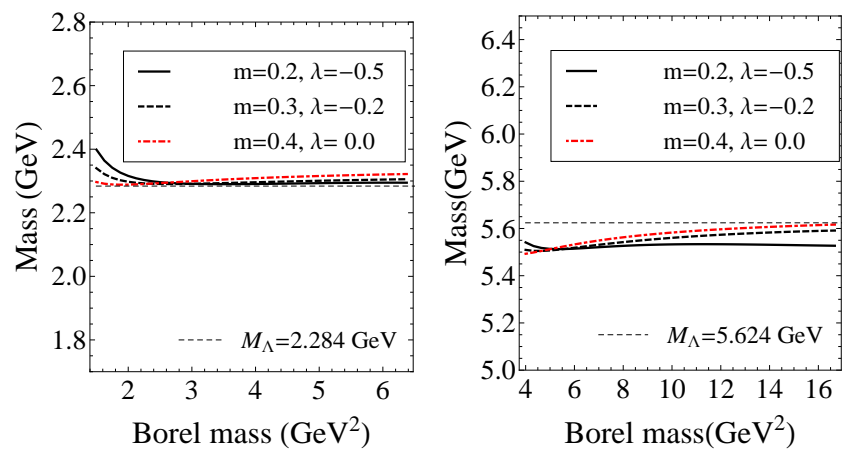

FIG. 3: (Color online) Borel curves for $\Lambda_{c}$ (left) and $\Lambda_{b}$ (right) with the good diquark parameters.

ing a very weak dependence on the higher dimensional operators.

\section{B. $\Lambda_{c}$ and $\Lambda_{b}$}

We also calculate the $\Lambda_{c}$ and $\Lambda_{b}$ masses using the good diquark parameters obtained in the $\Lambda$ sum rule, we set $s_{0}=7$ and $40 \mathrm{GeV}^{2}$, respectively. Figure 3 shows the Borel curves for $\Lambda_{c}$ and $\Lambda_{b}$ obtained with the sum rule given in Eq. (10). As can be seen in the figure, all sets of parameters $\left(\lambda, m_{\phi}\right)$ that worked well for $\Lambda$ reproduce the observed $\Lambda_{c}$ very well. Although the best Borel curve slightly underestimates the mass of $\Lambda_{b}$, overall our results imply that the diquark description works well again, suggesting that, in these $\Lambda_{h}$ baryons, the diquark configuration represents important degrees of freedom having universal properties.

In Fig. 4, we show the stability in the choice of the continuum threshold by plotting the Borel curve for different $s_{0}$ values. Here, we plot the graphs for one set of the accepted value of $\left(\lambda, m_{\phi}\right)=(-0.5,0.2 \mathrm{GeV})$. As can be seen from the figure, we obtain a reasonable mass of $\Lambda_{c}$ when choosing the value of $s_{0}$ which gives the most stable Borel curve. Unfortunately, the threshold parameter that gives the most stable Borel curve slightly underestimates the mass of $\Lambda_{b}$. We believe that the large difference in the bottom quark mass requires the use of different renormalization points for the vacuum expectation values, which can be a topic of future study.

\section{Summary and discussions}

The calculation of the correlation function as given in Eq. (1) means performing an OPE, assuming that the diquark structure is intact even when the relative momentum between the diquark effective field and the heavy quark is large. This is a simplification, but since we are interested only in the structure of the physical $\Lambda$, all we need is that the approximation be valid for the physical 
$\Lambda, \Lambda_{c}$ and $\Lambda_{b}$, as has been shown through our QCD sum rule analysis.

On the other hand, when we are analyzing states that have another light quark or an antiquark, the diquark description is expected to fail. For example, in the proton, the $u d$ diquark, despite its strong correlation, will tunnel into a new $d u$ diquark with the spectator $u$ quark. In our language, we would need an effective $\phi-u-d$ coupling to include this process. The actual application of our sum rule to the nucleon case obtained by taking $m_{s}$ to zero gives a nucleon mass above $1 \mathrm{GeV}$ without a stable Borel curve. In that sense the minimal Lagrangian for the diquark given in Eq. (2) is unique for describing $\Lambda_{h}$.

We have also analyzed the sum rule with a $J_{\sigma}=\phi^{\dagger} \phi$ current. In the scalar nonet picture, the $\sigma$ meson is composed of four quarks, but will have a strong quarkantiquark component. So we expect the coupling of our current with the physical $\sigma$ to be very small. Indeed, we find that the sum rule obtained does not reproduce the $\sigma$ mass around 400 to $600 \mathrm{MeV}$. Instead we find a monotonically decreasing Borel curve where the mass ranges from 1400 to $1000 \mathrm{GeV}$. The explicit treatment of four quark fields in the QCD sum rule has reproduced a lower mass with a stable curve [25].

In conclusion, we have introduced an explicit diquark degree of freedom and analyzed the sum rule for $\Lambda, \Lambda_{c}$ and $\Lambda_{b}$. We find that we can reproduce the masses of these states well, suggesting that these states can be represented by a strong diquark and a spectator quark. We further find that there is a duality between diquark con- densate and diquark mass from which we can estimate the constituent diquark mass to be around $400 \mathrm{MeV}$. We expect that such an explicit diquark degree of freedom can be an effective method to describe configurations where diquark correlation is expected to remain strong.

Acknowledgments: This work was supported by the Korean Ministry of Education through the BK21 Program and Project No. KRF-2006-C00011. The work of D.J. is supported by the Grant for Scientific Research No. 22105507 and No. 22740161 from MEXT of Japan. A part of this work was done in the Yukawa International
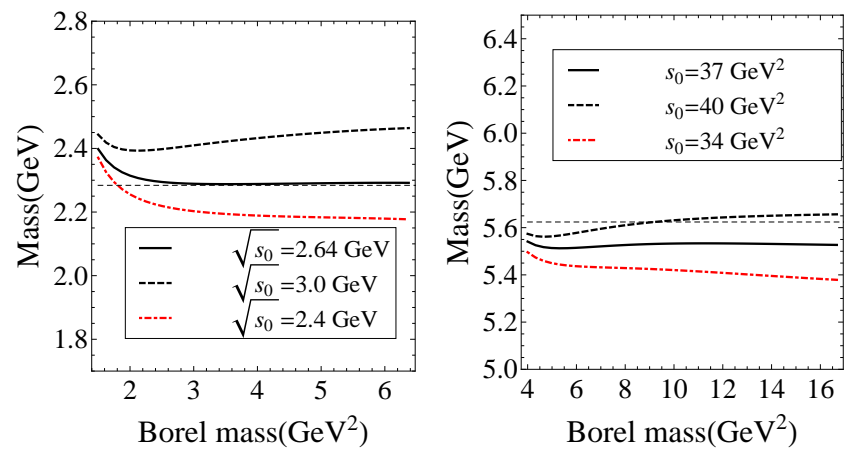

FIG. 4: (Color online) Borel curves showing the different choices of $\sqrt{s_{0}}$ for $\Lambda_{c}$ (left) and $\Lambda_{b}$ (right) with the solid lines showing the case with the chosen thresholds.

Project for Quark-Hadron Sciences (YIPQS).
[1] M. Ida and R. Kobayashi, Prog. Theor. Phys. 36, 846 (1966).

[2] D. B. Lichtenberg and L. J. Tassie, Phys. Rev. 155, 1601 (1967).

[3] R. L. Jaffe, Phys. Rept. 409, 1 (2005) [Nucl. Phys. Proc. Suppl. 142, 343 (2005)]

[4] M. Hess, F. Karsch, E. Laermann and I. Wetzorke, Phys. Rev. D 58, 111502 (1998)

[5] R. Babich et al. J. High Energy Phys. 01 (2006) 086.

[6] K. Orginos, PoS LAT2005, 054 (2006).

[7] C. Alexandrou, P. de Forcrand, and B. Lucini, Phys. Rev. Lett. 97, 222002 (2006).

[8] R. L. Jaffe, Phys. Rev. D 15, 267 (1977); 281 (1977).

[9] M. G. Alford, K. Rajagopal and F. Wilczek, Phys. Lett. B 422, 247 (1998)

[10] R. Rapp, T. Schafer, E. V. Shuryak and M. Velkovsky, Phys. Rev. Lett. 81, 53 (1998)

[11] J. L. Rosner, Phys. Rev. D 75, 013009 (2007).

[12] A. Selem and F. Wilczek, arXiv:hep-ph/0602128

[13] H. J. Lipkin, Phys. Lett. B 195, 484 (1987)

[14] S. H. Lee, S. Yasui, W. Liu and C. M. Ko, Eur. Phys. J.
C 54, 259 (2008)

[15] S. H. Lee and S. Yasui, Eur. Phys. J. C 64, 283 (2009)

[16] M. A. Shifman, A. I. Vainshtein and V. I. Zakharov, Nucl. Phys. B 147, 385 (1979).

[17] M. A. Shifman, A. I. Vainshtein and V. I. Zakharov, Nucl. Phys. B 147, 448 (1979).

[18] B. L. Ioffe, Z. Phys. C 18, 67 (1983).

[19] L.J. Reinders, H. Rubinstein and S. Yazaki, Phys. Rept. 127, 1 (1985).

[20] T. Hatsuda, Y. Koike and S. H. Lee, Nucl. Phys. B 394, 221 (1993).

[21] F. Klingl, N. Kaiser and W. Weise, Nucl. Phys. A 624, 527 (1997).

[22] T. Doi, Y. Kondo and M. Oka, Phys. Rept. 398, 253 (2004).

[23] E. Bagan, M. Chabab, H. G. Dosch and S. Narison, Phys. Lett. B 278, 367 (1992).

[24] S. Narrison, QCD as a Theory of Hadrons (Cambridge University Press, Cambridge, 2004)

[25] T. Kojo, D. Jido, Phys. Rev. D78, 114005 (2008). 